\title{
Improving the Recommendation of Mobile Services by Interpreting the User's Icon Arrangement
}

\author{
Matthias Böhmer \\ Münster University of Applied Sciences \\ Lab for Software Engineering, Germany \\ matthias.boehmer@fh-muenster.de
}

\author{
Gernot Bauer \\ Münster University of Applied Sciences \\ Lab for Software Engineering, Germany \\ gernot.bauer@fh-muenster.de
}

\begin{abstract}
The aether soon will be pervaded with a high density of digital services for usage on mobile phones. Personalization plays a crucial role for the success and acceptance of such systems. In this paper, we present work in progress on a new approach for improving the personalization of recommender systems for pervasive services. Mobile utilization is extended from services inherent in devices to pervasive services. We describe a new concept for collaborative and content-based filtering based on the users' service rankings given by arrangement of menu icons and discuss different models for interaction with a varying icon menu.
\end{abstract}

\section{Categories and Subject Descriptors}

H.3.3 [Information Search and Retrieval]: Information filtering, relevance feedback

\section{General Terms}

Design, Human Factors.

\section{Keywords}

Personalization, context-awareness, touch-based interaction.

\section{INTRODUCTION \& RELATED WORK}

In the nearby future, our environment will face us with the problem of choice overload for mobile services. The growth of a pervasive service environment is supported by emerging technologies in mobile and ubiquitous computing. Furthermore, many approaches today already propose methods and realize systems for embedding services into our everyday environment $[2,3]$.There are boundless possibilities: information delivery (e.g. news, weather), mobile guides (e.g. for cities or museums), communication services (e.g. presence, messaging), advertising (e.g. coupons), domotics (e.g. health care), or even user-generated content.

Context-aware systems seek out the subset of individual and situational relevant services for an end-user. They try

Copyright is held by the author/owner(s).

MobileHCI'09, September 15 - 18, 2009, Bonn, Germany.

ACM 978-1-60558-281-8. to reason high-level context information (e.g. activities like brushing teeth) from low-level context features like location, acceleration, temperature, audio, or movement. Figuring out the user's context, it can be matched against available services. But the user's cognitive decision on the relevance of a service cannot be formalized and estimated a priori. Currently, there is a lack of accuracy in the recommendation of services, mainly due to uncertainty in modeling human behavior and changing interests.

To maximize the ease of use for context-aware services, there is the following dilemma: the users' interests must be taken into account, but explicitly stating them would be an additional burden. In this paper, we present a new way for sharpening the service recommendation by learning from the user's device interaction. Thereby, we leave the unanswerable question for the relevance of services to the specialist - i.e. the user himself. The contribution of this paper is a new approach for improving recommender systems for mobile services utilizing the feedback given by users through the touch-based arrangement of icons.

For example, when many people put the time schedule provided by the service of a train station to the foremost position in their smart phone menus, this might also be a beneficial recommendation for people who have never been to this station before. Further, if one specific user puts the city guiding service to the top position in every city he visits, this should also be the case in a city where he has never been before.

\section{BEYOND FIXED SERVICE MENUS}

Figure 1 shows four different cases (C1-C4) how services can be supported by mobile devices. In one direction, the matrix differentiates between a fixed set of services and a set of services varying depending on the users location. Along the other axis it distinguishes whether the user-given arrangement of the services is related to location or not.

In C1 every user has a fixed set of icons on his phone, each one representing a service. He can arrange them for personal convenience, since the interaction on touch-enabled devices is very simple and intuitive. The user just has to drag the icon by touching it and drop it at the desired place. Since this provides a good cost benefit relation, we may assume that users arrange the icons in the menu according to their personal relevance of the services. For example, they will put the most used - and therefore most relevant - icon to the foremost position on screen. Thus, the relevance of a service can be derived from the icon arrangement.

In $\mathrm{C} 2$ the user-given arrangement of the icons becomes 


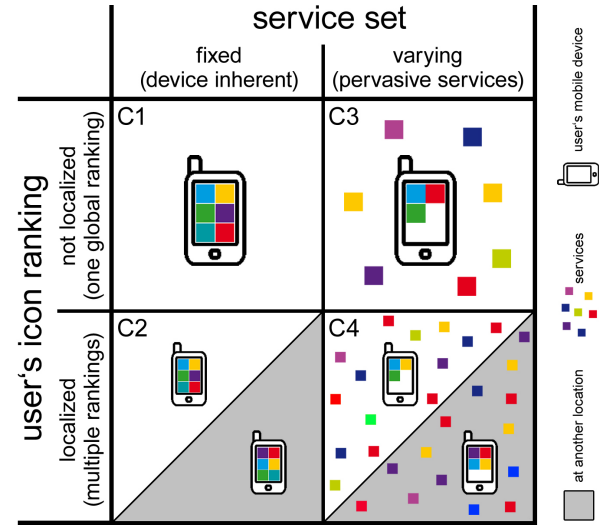

Figure 1: Different cases for service and icon handling on mobile phones.

localized, while the functionality provided by the device as a set of services stays fixed. The menu is able to adapt the icon arrangement according to location, when the user is on the move. This is similar to defining localized device profiles: e.g. another ring tone at home than at office.

Mobile phones are personal proxies, through which the user can interact with his environment [4]. Since the user's tasks and needs are constantly changing, his requirements concerning the interface also change. Therefore, it has to adapt to the environment. Accordingly, the set of relevant services will vary. Another reason for introducing a varying service set is the high density the environment will be pervaded with. Not all functionality is reasonable to a user in situ, because the small screen would be flooded. Therefore, only a selective subset of all available services should be presented.

C3 is more or less only theoretical. The user will never be able to define a ranking for all services, since he will never have them all on the menu at once. Further, in this case the benefit of the location-aware adaptive menu is not given.

C4 suggests multiple localized arrangements of the icons and the provision of a varying service set. While the user is able to adapt the menu to his needs, the device itself is adaptive and provides services in a location-aware manner. The main difference between $\mathrm{C} 1 / \mathrm{C} 2$ and $\mathrm{C} 3 / \mathrm{C} 4$ is that in case of a varying service set, the services are available depending on the user's environment and the menu becomes adaptive to this, whereas in case of a fixed service set, the services are always there, whether relevant or not.

The feedback of the user-given ranking of services can be included into existing context-aware recommender systems, especially for collaborative filtering, if a large group of users is under surveillance.

\section{INTERACTION DESIGN}

We establish a direct interaction between users and recommender systems. The decision on the services' relevance determined by the user's mental process is directly announced via touch-based interaction. Following a mixed-initiative approach [1], on the one side of the device's interface, the user interacts with the icons and adapts the menu to his current needs, whereas on the other side, the system evaluates the set of relevant services and injects their icons into the main menu of the user's device. This adaptation requires further refinements in order to avoid misinterpretations and user confusion.

There are different approaches for mapping the relevance of an icon to the display, e.g. by adjusting size or color intensity. In our case, the relevance of an icon is naturally given by its screen position, which can simply be manipulated.

For the arrangement of icons, the user can drag one icon and drop it to the desired place. The corresponding service gets a new relevance related to the position of the icons of the other services.

The recommender system injects services into the user's menu. Each service carries an estimated relevance relative to the other services. The recommender system has to know where to position the icon corresponding to a new service.

Devices using a joystick for navigation are often highlighting an icon, and menus have an initial focus, e.g. in the center of the screen. This focus point can be seen as the most relevant icon position, since it catches the user's attention subconsciously. Touch-based devices have no cursor, since every icon can be reached without navigation. Therefore no icon position is emphasized a priori.

A text-like mapping is obvious, since its mental model is based on text reading as daily activity. Other mappings can be designed according to ergonomic aspects. In case of a onehanded thumb-based usage of the device the icons should be mapped by reachability. With a high support of motor memory in the arrangement pattern, such an ergonomic mapping makes sense.

\section{CONCLUSIONS AND FUTURE WORK}

In this paper, we presented a new approach for improving the personalization of recommender systems for pervasive services. The main menu was extended from showing a fixed and device inherent set of services to a varying set of services provided in the environment of a user. We focused on an mixed-initiative approach for sharpening the recommendation of mobile services. The user-given arrangement of the icons in the main menu can be used as relevance input for the estimation of situational and personal considerable services. The user's interaction with the main menu of a mobile device stays simple, but becomes valuable for the location-awareness of collaborative and content-based filtering.

An extension of the presented location-based arrangement might base on a wider notion of context. Such as a spatiotemporal icon arrangement might bring an additional benefit to the user experience of pervasive services. For example, when somebody at home and in the evening always puts the icon of the alarm clock in the foremost menu position (to set the alarm), and in the morning he wants the icon for the bus schedule at the same position, an intelligent behavior adopting to this icon arrangement might be beneficial.

\section{REFERENCES}

[1] E. Horvitz. Principles of mixed-initiative user interfaces. In Proc. CHI '99, 159-166, 1999.

[2] R. Hull, B. Clayton, and T. Melamed. Rapid authoring of mediascapes. In Proc. UbiComp '04, 125-142, 2004.

[3] J. Krösche and S. Boll. The xpoi concept. In Proc. LOCA '05, 113-119. 2005.

[4] R. Want. You are your cell phone. Pervasive Computing, IEEE, 7(2):2-4, 2008. 